International Journal of Pure and Applied Mathematics

Volume 88 No. $3 \quad 2013,443-463$

ISSN: 1311-8080 (printed version); ISSN: 1314-3395 (on-line version)

url: http://www.ijpam.eu

doi: http://dx.doi.org/10.12732/ijpam.v88i3.12

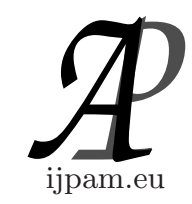

\title{
THE RIEMANN HYPOTHESIS IS JUSTIFIED BY THREE DIFFERENT PROOF WAYS
}

\author{
Hao-Cong $\mathrm{Wu}$
}

Room 2401, Block F, Mingrun Garden Buildings

No. 27 W. Jiefang Road, Zhanjiang City

Guangdong Province 524000, P.R. CHINA

\begin{abstract}
Using the integral convergence attaching analytic properties as well as the symmetry of the nontrivial zeros of the Riemann zeta function all prove the Riemann Hypothesis and using topological method also proves it.
\end{abstract}

AMS Subject Classification: 11M26, 46A63

Key Words: Laplace transform, converges uniformly, differentiable, analytic, Riemann Zeta functional equations, homeomorphism, open neighborhoods, boundary points, contractible

\section{The First Proof from The Integral Convergence}

\subsection{Introduction}

We show how the convergence of the integral

$$
\int_{1}^{\infty} \frac{\log x}{x^{\frac{3}{2}+\varepsilon}} d x
$$

proves the function

$$
g(z)=\int_{1}^{\infty} \frac{|\psi(x)-x|}{x^{z+2-\left(\lambda_{0}-\lambda\right)}} d x
$$

is differentiable for $\operatorname{Re}(z) \geq 0$, with $\psi(x)=x+O\left(x^{\lambda}\right)$ for $\lambda \geq \frac{1}{2}$, and $\lambda_{0}-\lambda \leq$ $-\frac{1}{2}-\varepsilon$ for $\forall \varepsilon>0$, where $\lambda_{0}$ is dependent of $\varepsilon$ and $\lambda$ can be independent of $\varepsilon$.

Received: September 3, 2013

(c) 2013 Academic Publications, Ltd. url: www.acadpubl.eu 
Let $\rho$ be all nontrivial zeros of $\zeta(s)$, we know $\psi(x)=x+O\left(x^{\sup R e(\rho)+\varepsilon}\right)$ for $\forall \varepsilon>0$. Indeed, if there is not a positive number lambda is less than 1 for the form $\psi(x)=x+O\left(x^{\lambda}\right)$, then by the definition of the supremum involving its two conditions, we get $\sup \operatorname{Re}(\rho)=\max \operatorname{Re}(\rho)$, and then we get another result is false that $\zeta(s)$ has a $\sup \operatorname{Re}(\rho) \geq 1$ leading to have a $\operatorname{Re}(\rho) \geq 1$ on the region $0<\operatorname{Re}(s)<1$ (the critical strip), which is a contradiction. So, there exists a positive number which a suitable lambda is less than 1 for the form $\psi(x)=x+O\left(x^{\lambda}\right)$.

In fact, we may use the condition of a suitable lambda is less than 1 for the form $\psi(x)=x+O\left(x^{\lambda}\right)$, but here we don't use it. In a nutshell, we have $\psi(x)=x+O\left(x^{\lambda}\right)$ for $\lambda \geq \frac{1}{2}$, and $\psi(x)=O(x)$.

Furthermore, using a basic fact from properties of the zeta function that $\zeta(s)$ certainly has an infinite number of the nontrivial zeros in the region $\operatorname{Re}(s) \geq \frac{1}{2}$ and the symmetry of the zeros such that we can surely get $\lambda \geq \frac{1}{2}$ for $\psi(x)=$ $x+O\left(x^{\lambda}\right)$,then we can conclude the integral

$$
\int_{1}^{\infty} \frac{\psi(x)-x}{x^{\frac{3}{2}+\varepsilon}} d x
$$

converges absolutely and uniformly for $\forall \varepsilon>0$, which concludes the integral

$$
\int_{1}^{\infty} \frac{\psi(x)-x}{x^{s+1}} d x
$$

is analytic for $\operatorname{Re}(s)>\frac{1}{2}$. Thus this concludes the proof of the form $\psi(x)=$ $x+O\left(x^{\frac{1}{2}+\varepsilon}\right)$ for $\forall \varepsilon>0$.

\subsection{Preliminaries}

\subsubsection{Basic Properties of the Zeta Function}

For $\operatorname{Re}(s)>1$ we know that the Riemann zeta function defined by the series

$$
\sum_{n=1}^{\infty} \frac{1}{n^{s}}
$$

and the Euler product

$$
\prod_{p}\left(1-p^{-s}\right)^{-1}
$$

namely

$$
\zeta(s)=\sum_{n=1}^{\infty} \frac{1}{n^{s}}=\prod_{p}\left(1-p^{-s}\right)^{-1} .
$$


The Euler product shows that $\zeta(s) \neq 0$ for $\operatorname{Re}(s)>1$. The series and the Euler product converge absolutely and uniformly for $\operatorname{Re}(s) \geq 1+\delta$, with any $\delta>0$. In fact,we often obtain the following identities, valid for $\operatorname{Re}(s)>1$ :

$$
\begin{gathered}
\zeta(s)=\sum_{n=1}^{\infty} n^{-s}=\sum_{n=1}^{\infty} s \int_{n}^{\infty} \frac{d x}{x^{s+1}}= \\
=s \int_{1}^{\infty}\left(\sum_{n \leq x} 1\right) \frac{d x}{x^{s+1}}=s \int_{1}^{\infty} \frac{[x]}{x^{s+1}} d x=\frac{s}{s-1}-s \int_{1}^{\infty} \frac{\{x\}}{x^{s+1}} d x .
\end{gathered}
$$

Also, for $\operatorname{Re}(s)>1$ we know the identity $(* !)$ :

$$
\begin{gathered}
\phi(s)=-\frac{\zeta^{\prime}(s)}{\zeta(s)}=\sum_{n=1}^{\infty} \frac{\Lambda(n)}{n^{s}}= \\
=\sum_{p} \frac{\log p}{p^{s}-1}=s \int_{1}^{\infty} \frac{\psi(x)}{x^{s+1}} d x=\frac{s}{s-1}+s \int_{1}^{\infty} \frac{\psi(x)-x}{x^{s+1}} d x,
\end{gathered}
$$

where the sum is

$$
\sum_{p} \frac{\log p}{p^{s}-1}
$$

extended over all primes. Moreover, we know the integral $\int_{1}^{\infty} \frac{\{x\}}{x^{s+1}} d x$ converges absolutely,and uniformly for $\operatorname{Re}(s) \geq \delta$ with any $\delta>0$. The symbol $[x]$ denotes the greatest integer $\leq x$, it is called the integral part of $x$, the number $\{x\}=$ $x-[x]$ is called the fractional part of $x$, it satisfies the inequalities $0 \leq\{x\}<1$, with $\{x\}=0$ if and only if $x$ is an integer.

We define

$$
\psi(x)=\sum_{p^{m} \leq x} \log p=\sum_{n \leq x} \Lambda(n) .
$$

The $\Lambda(n)$ is $\log p$ when $\exists m \geq 1$ such that $n=p^{m}$, or otherwise it is 0 . Where the sum is taken over those integers of the form $p^{m}$ that are less than or equal to $x$. Here $p$ is a prime number and $m$ is a positive integer. Moreover, we see that $\zeta(1+i t) \neq 0, \zeta(i t) \neq 0$, and when the function $\zeta^{\prime} / \zeta(s)$ has no poles on the region $1>\operatorname{Re}(s)>\frac{1}{2}$, then which implies that the function $\zeta(s)$ has no zeros on the region $1>\operatorname{Re}(s)>\frac{1}{2}$.

We define

$$
\Phi(s)=\sum_{p} \frac{\log p}{p^{s}}
$$


for $\operatorname{Re}(s)>1$. Here $p$ is a prime number. The sum defining $\Phi(s)$ converges uniformly and absolutely for $\operatorname{Re}(s) \geq 1+\delta$, by the same argument as for the sum defining the zeta function, which one sees the estimating $\left|\frac{1}{n^{s}}\right| \leq \frac{1}{n^{1+\delta}}$ and the series $\sum \frac{1}{n^{1+\delta}}$ converges absolutely and uniformly for any $\delta>0$.

Since

$$
e^{x}=1+x+\ldots+\frac{x^{n}}{n !}+\frac{x^{n+1}}{(n+1) !}+\ldots
$$

it follows that

$$
x^{-n} e^{x}>\frac{x}{(n+1) !}
$$

for positive $x$ and for all $n$. For any fixed $n$, as $x \rightarrow \infty$, it follows that $e^{x}$ grows faster than any fixed power of $x$. We can write $x^{n}=o\left(e^{x}\right)$ to mean

$$
\lim _{x \rightarrow \infty} \frac{x^{n}}{e^{x}}=0
$$

for all $n$. Getting $\log x=o\left(x^{\delta}\right), \delta>0$. Then as above the sum $\Phi(s)$ we merely use the fact that given $\delta>0, \log n \leq n^{\varepsilon}$ for all $n \geq n_{0}(\varepsilon)$.

\subsubsection{Basic Results}

In a way, we need go through the basic results. We recall the notation: $f=O(g)$ mean that $f, g$ are two functions of a variable $x$, defined for all $x$ sufficiently large, and $g$ is positive, there exists a constant $C>0$ such that $|f(x)| \leq C g(x)$ for all $x$ sufficiently large. We also recall the results, let $\rho$ be all nontrivial zeros of $\zeta(s)$, we know $\psi(x)=x+O\left(x^{\sup R e(\rho)+\varepsilon}\right)$ for every $\varepsilon>0$. Intimately, we can get $\lambda \geq \operatorname{supRe}(\rho)+\varepsilon$ by the result that $\zeta(s)$ has no zero on the region $\operatorname{Re}(s)>\lambda$. Using a basic fact from properties of the zeta function that $\zeta(s)$ certainly has an infinite number of the nontrivial zeros in the region $\operatorname{Re}(s) \geq \frac{1}{2}$ and the symmetry of the nontrivial zeros such that we have $\lambda \geq \frac{1}{2}$. We know that there exists a positive number which a suitable lambda is less than 1 for the form $\psi(x)=x+O\left(x^{\lambda}\right)$. In a nutshell, we have $\psi(x)=x+O\left(x^{\lambda}\right)$ for $\lambda \geq \frac{1}{2}$, and $\psi(x)=O(x)$.

\subsection{The Basic Theorem}

Theorem 1. The function $\Phi$ is meromorphic for $\operatorname{Re}(s)>\frac{1}{2}$. Furthermore, for $\operatorname{Re}(s) \geq 1$, we have $\zeta(s) \neq 0$ and $\Phi(s)-\frac{1}{s-1}$ has no poles for $\operatorname{Re}(s) \geq 1$. 
Proof. For $\operatorname{Re}(s)>1$, the Euler product shows that $\zeta(s) \neq 0$. We know the result

$$
-\zeta^{\prime} / \zeta(s)=\sum_{p} \frac{\log p}{p^{s}-1}
$$

for $\operatorname{Re}(s)>1$, and the identity $(* !)$ :

$$
\phi(s)=-\zeta^{\prime} / \zeta(s)=\frac{s}{s-1}+s \int_{1}^{\infty} \frac{\psi(x)-x}{x^{s+1}} d x
$$

for $\operatorname{Re}(s)>1$. Using the geometric series we get the expansion

$$
\frac{1}{p^{s}-1}=\frac{1}{p^{s}} \times \frac{1}{1-\frac{1}{p^{s}}}=\frac{1}{p^{s}}\left(1+\frac{1}{p^{s}}+\frac{1}{p^{2 s}}+\ldots\right)=\frac{1}{p^{s}}+\frac{1}{p^{2 s}}+\ldots
$$

So the identity $(* !)$ satisfies

$$
-\zeta^{\prime} / \zeta(s)=\Phi(s)+\sum_{p} h_{p}(s)
$$

where $\left|h_{p}(s)\right| \leq B \frac{\log p}{\left|p^{2 s}\right|}$ for some constant $B$. But the series $\sum \frac{\log n}{n^{2 s}}$ converges absolutely and uniformly for $\operatorname{Re}(s) \geq \frac{1}{2}+\delta$, with any $\delta>0$, and we know the intimate result that the function $\zeta(s)-\frac{1}{s-1}$ extends to an analytic function on the region $\operatorname{Re}(s)>0$, this result and the identity $(* !)$ imply that $\Phi$ is meromorphic for $\operatorname{Re}(s)>\frac{1}{2}$, and has a pole at $s=1$ and at the zeros of $\zeta$, but no other poles in this region. We omit the proof that $\zeta$ has no zero on the line $\operatorname{Re}(s)=1$, which is just the intimate result. This proves the theorem.

\subsection{The Main Lemmas}

\subsubsection{Stating Some Basic Conditions}

We know that the form $\psi(x)=x+O\left(x^{\frac{1}{2}+\varepsilon}\right)$ for $\forall \varepsilon>0$ implies the Riemann Hypothesis. We shall now prove the main lemmas, which constitute the delicate part of the proof.

A function $f$ of two variables, $f(t, z)$, where $z$ lies in some domain $U$ in the complex numbers plane, and we let $D_{1} f$ and $D_{2} f$ be the partial derivatives of $f$ with respect to the first and second variable respectively. We know the integral

$$
\int_{0}^{\infty} f(t, z) d t=\lim _{B \rightarrow \infty} \int_{0}^{B} f(t, z) d t
$$


is said to be uniformly convergent for $z \in U$, if and only if, given $\varepsilon$, there exists $B_{0}$ such that $B_{0}<B_{1}<B_{2}$, i.e., select $B_{1}$ so sufficiently large, then

$$
\left|\int_{B_{1}}^{B_{2}} f(t, z) d t\right|<\varepsilon .
$$

The integral is absolutely and uniformly convergent for $z \in U$ if the same condition holds with $f(t, z)$ replaced by the absolute value $|f(t, z)|$.

In a way, if the absolute value $|f(t, z)|$ is decreasing for all sufficiently large $t$, we can then use the inequality $\left|\int_{B_{1}}^{B_{2}} f(t, z) d t\right|<\varepsilon$, or $\left|\int_{n}^{n+1} f(t, z) d t\right|<\varepsilon$, and select $B_{1}=n$ so sufficiently large and $a \geq 0$ to know the integral

$$
\int_{a}^{\infty} f(t, z) d t=\lim _{b \rightarrow \infty} \int_{a}^{b} f(t, z) d t
$$

converges uniformly. For given $\varepsilon>0$, there exists $B_{0} \geq 1$ and such that $B_{0}<B_{1}=n<n+1 \leq B_{2}$, and select $B_{1}$ so sufficiently large, obviously we get

$$
\int_{B_{1}}^{B_{2}} \frac{\log x}{x^{\frac{3}{2}+\varepsilon}} d x \rightarrow 0
$$

being $\log x=o\left(x^{\delta}\right), \delta>0$ and

$$
\int_{n}^{n+1} \frac{\log x}{x^{\frac{3}{2}+\varepsilon}} d x \rightarrow 0 .
$$

We can write the expression under the integral sign as $\frac{\log x}{x^{\frac{3}{2}+\varepsilon}}$, where $\forall \varepsilon>0$ can be independent of $x$. Given any compact subset $\mathrm{K}$ of the region defined by $x \geq 1$, we note that $\frac{\log x}{x^{\frac{3}{2}+\varepsilon}} \rightarrow 0$ rapidly uniformly for $x \in K$ as $x \rightarrow \infty$. The word "rapidly" means that this $x^{\frac{3}{2}+\varepsilon}$ divided by this $\log x$ tends to 0 uniformly for $x$ in $K$ as $x \rightarrow \infty$.

Then, obviously the integral

$$
\int_{1}^{\infty} \frac{\log x}{x^{\frac{3}{2}+\varepsilon}} d x
$$

is convergent for $\forall \varepsilon>0$. Using the basic fact that the integral $\int_{1}^{\infty} \frac{d x}{x^{n}}$ converges absolutely for any $n>1$, we know the integral

$$
\int_{1}^{\infty} \frac{d x}{x^{\frac{3}{2}+\varepsilon}}
$$

is also convergent for $\forall \varepsilon>0$. 


\subsubsection{The Main Lemmas for the Application}

Lemma 2. (The Differentiation Lemma ) Let $I$ be an interval of real numbers, possibly infinite. Let $U$ be an open set of complex numbers. Let $f=f(t, z)$ be a continuous function on $I \times U$. Assume:

(i) For each compact subset $K$ of $U$, the integral $\int_{I} f(t, z) d t$ is uniformly convergent for $z \in K$.

(ii) For each $t$ the function $z \mapsto f(t, z)$ is analytic.

Let $F(z)=\int_{I} f(t, z) d t$, then $F$ is analytic on $U, D_{2} f(t, z)$ satisfies the same assumptions as $f$, and $F^{\prime}(z)=\int_{I} D_{2} f(t, z) d t$.

(no proof given here, see, for instance, [1], p.409)

Lemma 3. (The Differentiation Lemma A1) Let $f$ be a function of two variables, $f(t, z)$, defined for $t \geq a$ and $z$ either $t \geq a$ in a finite interval $[c, d]$, or on some open set $U$ in $C$. Assume that $D_{2} f$ exists, and that both $f$ and $D_{2} f$ are continuous. Assume that there are functions $\varphi(t)$ and $\vartheta(t)$ which are $\geq 0$, such that $|f(t, z)| \leq \varphi(t)$ and $\left|D_{2} f(t, z)\right| \leq \vartheta(t)$, for all $t, z$, and such that the integrals $\int_{a}^{\infty} \varphi(t) d t$ and $\int_{a}^{\infty} \vartheta(t) d t$ converge. Let

$$
g(z)=\int_{a}^{\infty} f(t, z) d t
$$

Then $g(z)$ is differentiable, and

$$
g^{\prime}(z)=\int_{a}^{\infty} D_{2} f(t, z) d t .
$$

(no proof given here, see, for instance, [1], pp.286-290)

Another, let $f$ be a continuous function on the real numbers $\geq 0$ and assume that there is constants $A, B$ such that $|f(t)| \leq A e^{B t}$ for all $t$ sufficiently large. Moreover, and assume for simplicity that $f$ is bounded, piecewise continuous, whence $B \leq 0$. What we prove will hold under much less restrictive conditions: instead of piecewise continuous,it would suffice to assume that the integral $\int_{a}^{b}|f(t)| d t$ exists for every pair of numbers $a, b \geq 0$. We shall associate to $f$ the Laplace transform defined by $g(z)=\int_{0}^{\infty} f(t) e^{-z t} d t$ for $\operatorname{Re}(z)>0$. We can then apply the differentiation lemma, whose proof applies to a function satisfying our conditions(bounded and piecewise continuous), and then we easily conclude that $g$ is analytic for $\operatorname{Re}(z)>0$.

We shall now state special cases of the following lemmas concerning differentiation under the integral sign which are sufficient for our applications. 
Lemma 4. (Main Lemma A1) Let $f$ be bounded, piecewise continuous on the real numbers $\geq 0$. Let $f$ the Laplace transform $g$ defined by $g(z)=$ $\int_{0}^{\infty} f(t) e^{-z t} d t$ for $\operatorname{Re}(z)>0$, then $g$ is analytic in the region $\operatorname{Re}(z)>0$. If $g$ extends to an analytic function for $\operatorname{Re}(z) \geq 0$, then $\int_{0}^{\infty} f(t) d t$ exists and is equal to $g(0)$.

(no proof given here, see, for instance, [1], pp.449-452; [5], pp.134-137)

We claim that it also concludes the integral

$$
\int_{1}^{\infty} \frac{\psi(x)-x}{x^{\frac{3}{2}+\varepsilon}} d x
$$

is convergent for $\forall \varepsilon>0$. Observe that the function $\psi$ is piecewise continuous. In fact, it is locally constant: there is no change in $\psi$ between prime numbers. The application of Main Lemma $A 1$ is to prove:

Lemma 5. (Main Lemma A2) The pair of integral

$$
\int_{1}^{\infty} \frac{\psi(x)-x}{x^{\frac{3}{2}+\varepsilon}} d x
$$

and

$$
\int_{1}^{\infty} \frac{|\psi(x)-x|}{x^{\frac{3}{2}+\varepsilon}} d x
$$

converge for $\forall \varepsilon>0$.

(proof given here, see, for instance, [5], pp.137-139)

Proof. Let

$$
f_{1}(t)=\frac{\psi\left(e^{t}\right)-e^{t}}{e^{\left(1-\left(\lambda_{0}-\lambda\right)\right) t}}
$$

and

$$
f_{2}(t)=\frac{\left|\psi\left(e^{t}\right)-e^{t}\right|}{e^{\left(1-\left(\lambda_{0}-\lambda\right)\right) t}}
$$

where $\lambda$ satisfies the conditions $\psi(x)=x+O\left(x^{\lambda}\right), \lambda \geq \frac{1}{2}$, given $\lambda_{0} \leq \lambda-\frac{1}{2}-\varepsilon$ for $\forall \varepsilon>0$, show that given any $\lambda_{0} \leq \lambda-\frac{1}{2}-\varepsilon$ satisfies $1-\left(\lambda_{0}-\lambda\right) \geq \frac{3}{2}+\varepsilon$ for $\forall \varepsilon>0$, of course including the case $1-\left(\lambda_{0}-\lambda\right)=\frac{3}{2}+\varepsilon$, where $x=e^{t}$ is not less than 1 , obviously $t$ is not less than 0 , they are of course continuous, and $\lambda_{0}$ is dependent of $\varepsilon, \lambda$ can be independent of $\varepsilon$. Then $f_{i}$ is certainly piecewise continuous, and is bounded by the formulas $\psi(x)=O(x)$ and $\psi(x)=x+O\left(x^{\lambda}\right)$ for $\lambda \geq \frac{1}{2}$, where $i=1,2$. 
Making the substitution $x=e^{t}$ in the desired integral, $d x=e^{t} d t$, where $\exp (t)$ is not less than 1 , we see that

$$
\int_{1}^{\infty} \frac{\psi(x)-x}{x^{\frac{3}{2}+\varepsilon}} d x=\int_{0}^{\infty} f_{1}(t) d t
$$

and

$$
\int_{1}^{\infty} \frac{|\psi(x)-x|}{x^{\frac{3}{2}+\varepsilon}} d x=\int_{0}^{\infty} f_{2}(t) d t
$$

Therefore these suffice to prove that the integrals on the right converge, it suffices to prove that the Laplace transform $g_{i}$ of $f_{i}$ is analytic for $\operatorname{Re}(z) \geq 0$, so we have to compute the Laplace transform $g_{i}$. We claim that in the case $i=1$,

$$
g_{1}(z)=\frac{\phi\left(z+1-\left(\lambda_{0}-\lambda\right)\right)}{z+1-\left(\lambda_{0}-\lambda\right)}-\frac{1}{z-\left(\lambda_{0}-\lambda\right)} .
$$

Once we have proved the formula, we can then apply Theorem 1 and the identity (*!) to conclude that $g_{1}(z)$ is analytic for $\operatorname{Re}(z) \geq 0$, thus concluding the part of the proof of Main Lemma $A 2$.

Now to compute $g_{i}(z)$ when $i=1$, we use the identity $(* !)$, we obtain

$$
\frac{\phi(s)}{s}-\frac{1}{s-1}=\int_{1}^{\infty} \frac{\psi(x)-x}{x^{s+1}} d x .
$$

Then

$$
\begin{gathered}
g_{1}(z)=\frac{\phi\left(z+1-\left(\lambda_{0}-\lambda\right)\right)}{z+1-\left(\lambda_{0}-\lambda\right)}-\frac{1}{z-\left(\lambda_{0}-\lambda\right)}= \\
=\int_{1}^{\infty} \frac{\psi(x)-x}{x^{z+2-\left(\lambda_{0}-\lambda\right)}} d x=\int_{0}^{\infty} \frac{\psi\left(e^{t}\right)-e^{t}}{e^{\left(z+2-\left(\lambda_{0}-\lambda\right)\right) t}} e^{t} d t=\int_{0}^{\infty} f_{1}(t) e^{-z t} d t,
\end{gathered}
$$

where $\operatorname{Re}\left(z+1-\left(\lambda_{0}-\lambda\right)\right)>1$ and so $\operatorname{Re}(z)>\lambda_{0}-\lambda$, we have $\lambda_{0}-\lambda \leq-\frac{1}{2}-\varepsilon$ for $\forall \varepsilon>0$, which by Theorem 1 and the identity $(* !)$ show that $g_{1}(z)$ is analytic for $\operatorname{Re}(z)>\lambda_{0}-\lambda$, and then it is analytic for $\operatorname{Re}(z) \geq 0$. This gives us the Laplace transform of $f_{1}$, and concludes the part of the proof of Main Lemma $A 2$ by Main Lemma $A 1$.

We claim that in the case $i=2$,

$$
\begin{gathered}
g_{2}(z)=\int_{0}^{\infty} \frac{\left|\psi\left(e^{t}\right)-e^{t}\right|}{e^{\left(z+2-\left(\lambda_{0}-\lambda\right) t\right.}} e^{t} d t= \\
=\int_{1}^{\infty} \frac{|\psi(x)-x|}{x^{z+2-\left(\lambda_{0}-\lambda\right)}} d x=\int_{0}^{\infty} f_{2}(t) e^{-z t} d t
\end{gathered}
$$


where $f_{2}(t)=\frac{\left|\psi\left(e^{t}\right)-e^{t}\right|}{e^{\left(1-\left(\lambda_{0}-\lambda\right) t\right.}}$, whence the function

$$
g(z)=\int_{1}^{\infty} \frac{|\psi(x)-x|}{x^{z+2-\left(\lambda_{0}-\lambda\right)}} d x
$$

converges absolutely for $\operatorname{Re}(z) \geq 0$ and uniformly for $\operatorname{Re}(z) \geq \delta$ with $\delta \geq 0$ from the basic fact that the integral $\int_{1}^{\infty} \frac{d x}{x^{n}}$ converges absolutely and uniformly for $n \geq 1+k$, with any $k>0$. Given any $\lambda_{0} \leq \lambda-\frac{1}{2}-\varepsilon$ for $\forall \varepsilon>0$, with $\psi(x)=x+O\left(x^{\lambda}\right), \lambda \geq \frac{1}{2}$, when $\operatorname{Re}(z) \geq 0$ we have $\operatorname{Re}(z)+2-\left(\lambda_{0}-\lambda\right)>2$, then by the differentiation lemma which show that $g_{i}(z)$ is analytic for $\operatorname{Re}(z) \geq 0$ when $i=2$, and this gives us the Laplace transform of $f_{2}$, and concludes the part of the proof of Main Lemma $A 2$ by Main Lemma $A 1$. Therefore, this proves the lemma.

We shall now recall that the function $g_{2}(z)$ is analytic for $\operatorname{Re}(z) \geq 0$ how it is proved by the differentiation lemma $A 1$. Also, in fact, the factor $e^{t}$ is determined by $x=e^{t}$ generated, which are sufficient in some conditions, it only considers the variable $x$ changes, which the corresponding variation of the variable $e^{t}$ should be negligible. Lets put the details as follows.

\subsubsection{The Assertion of the Integral Convergence Concludes Main Lemma A2}

The assertion of the convergence of the integral

$$
\int_{1}^{\infty} \frac{|\psi(x)-x|}{x^{\frac{3}{2}+\varepsilon}} d x
$$

is also concluded by the convergence of the integral

$$
\int_{1}^{\infty} \frac{\log x}{x^{\frac{3}{2}+\varepsilon}} d x
$$

for all $x$ so sufficiently large. From now on, we can apply the differentiation lemma $A 1$, whose proof applies to the function

$$
g_{2}(z)=\int_{1}^{\infty} \frac{|\psi(x)-x|}{x^{z+2-\left(\lambda_{0}-\lambda\right)}} d x
$$

for $\operatorname{Re}(z) \geq 0$ satisfying our conditions:

(1) $\psi(x)=x+O\left(x^{\lambda}\right), \lambda \geq \frac{1}{2}, \lambda_{0}-\lambda \leq-\frac{1}{2}-\varepsilon$ for $\forall \varepsilon>0$. 
(2) For all $x$ so sufficiently large, we have the inequality

$$
\begin{gathered}
\left|\frac{|\psi(x)-x|}{x^{z+2-\left(\lambda_{0}-\lambda\right)}}\right|=\frac{|\psi(x)-x|}{\left|x^{z+2-\left(\lambda_{0}-\lambda\right)}\right|} \leq \\
\leq\left|\frac{B x^{\lambda}}{x^{2-\left(\lambda_{0}-\lambda\right)}}\right| \leq\left|\frac{B}{x^{2-\lambda_{0}}}\right| \leq B\left|\frac{1}{x^{\frac{3}{2}+\varepsilon}}\right|=\varphi(x),
\end{gathered}
$$

where $B$ is some constant and $x \geq 1$.

(3) Obviously,for $x \geq 1$, we see that $f=\frac{|\psi(x)-x|}{x^{z+2-\left(\lambda_{0}-\lambda\right)}}, D_{2} f=D_{2}\left(\frac{|\psi(x)-x|}{x^{z+2-\left(\lambda_{0}-\lambda\right)}}\right)$ exists, and holds for all $x$ so sufficiently large, and that both $f$ and $D_{2} f$ are continuous. For all $x$ so sufficiently large,we have the inequality

$$
\begin{aligned}
& \left|D_{2}\left(\frac{|\psi(x)-x|}{x^{z+2-\left(\lambda_{0}-\lambda\right)}}\right)\right|=\left|\frac{|\psi(x)-x| \cdot(-\log x)}{e^{\left(z+2-\left(\lambda_{0}-\lambda\right)\right) \log x}}\right|= \\
& =\left|\frac{|\psi(x)-x| \cdot \log x}{x^{R e(z)+2-\left(\lambda_{0}-\lambda\right)}}\right| \leq\left|\frac{B \cdot \log x}{x^{\frac{3}{2}+\varepsilon}}\right|=B \frac{\log x}{x^{\frac{3}{2}+\varepsilon}}=\vartheta(x) .
\end{aligned}
$$

(4) Very easily, for all $x, z$,we know the integrals $\int_{1}^{\infty} \varphi(x) d x=B \int_{1}^{\infty} \frac{d x}{x^{\frac{3}{2}+\varepsilon}}$ and $\int_{1}^{\infty} \vartheta(x) d x=B \int_{1}^{\infty} \frac{\log x}{x^{\frac{3}{2}+\varepsilon}} d x$ converge from the integrals $\int_{1}^{\infty} \frac{d x}{x^{\frac{3}{2}+\varepsilon}}$ and $\int_{1}^{\infty} \frac{\log x}{x^{\frac{3}{2}+\varepsilon}} d x$ converge for all $x$ so sufficiently large and for $\forall \varepsilon>0$, where $B$ is some constant.Then by the differentiation lemma $A 1$, we get $g_{2}(z)$ is analytic for $\operatorname{Re}(z) \geq 0$. This gives us the Laplace transform of $f_{2}$ and concludes the part of the proof of Main Lemma $A 2$ by Main Lemma $A 1$.

Thus this puts the details as above, and this also proves the lemma.

\subsection{Conclusions from the Main Lemmas}

Using Main Lemma $A 2$ concludes that the integral

$$
\int_{1}^{\infty} \frac{\psi(x)-x}{x^{s+1}} d x
$$

converges absolutely and uniformly for $\operatorname{Re}(s)>\frac{1}{2}$, and by the differentiation lemma concludes the integral

$$
\int_{1}^{\infty} \frac{\psi(x)-x}{x^{s+1}} d x
$$


is analytic for $\operatorname{Re}(s)>\frac{1}{2}$, which immediately follows that the function $\zeta^{\prime} / \zeta(s)$ has no poles on the region $1>\operatorname{Re}(s)>\frac{1}{2}$ from the formula $(* !)$, and which implies that the function $\zeta(s)$ has no zeros on the region $1>\operatorname{Re}(s)>\frac{1}{2}$, thus concluding the proof of the form $\psi(x)=x+O\left(x^{\frac{1}{2}+\varepsilon}\right)$ for $\forall \varepsilon>0$, and it also concludes the proof of the Riemann Hypothesis.

\section{The Second Proof from the Symmetrically Located Nontrivial Zeros}

\subsection{Some Results for Using}

To establish the following Theorem 6, we first note that a complex number $z_{0}$ is a zero for the analytic function $f$ if $f\left(z_{0}\right)=0$. In particular, analytic continuation shows that the zeros of a nontrivial analytic function are isolated. In other words, if $f(z)$ is analytic in $\Omega$ and $f\left(z_{0}\right)=0$ for some $z_{0} \in \Omega$, then there exists an open neighborhood $U$ of $z_{0}$ such that $f\left(z_{0}\right) \neq 0$ for all $z \in U-\left\{z_{0}\right\}$, unless $f(z)$ is identically zero. We start with a local description of an analytic function near a zero.

Theorem 6. Suppose that $f(z)$ is analytic in a connected open set $\Omega$, has a zero at a point $z_{0} \in \Omega$, and does not vanish identically in $\Omega$. Then there exists a neighborhood $U \subset \Omega$ of $z_{0}$, a non-vanishing analytic function on $U$, and a unique positive integer $n$ such that $f(z)=\left(z-z_{0}\right)^{n} g(z)$ for all $z \in \Omega$.

Proof. Since $\Omega$ is connected and $f(z)$ is not identically zero, we conclude that $f(z)$ is not identically zero in a neighborhood of $z_{0}$. In a small disc centered at $z_{0}$ the function $f(z)$ has a power series expansion $f(z)=\sum_{k=0}^{\infty} a_{k}\left(z-z_{0}\right)^{k}$. Since $f(z)$ is not identically zero near $z_{0}$, there exists a smallest integer $n$ such that $a_{n} \neq 0$. Then, we can write $f(z)=\left(z-z_{0}\right)^{n}\left[a_{n}+a_{n+1}\left(z-z_{0}\right)+\cdots\right]=$ $\left(z-z_{0}\right)^{n} g(z)$, where $g$ is defined by the series in brackets, and hence is analytic,and is nowhere vanishing for all $z$ close to $z_{0}$ (since $a_{n} \neq 0$ ). To prove the uniqueness of the integer $n$, suppose that we can also write $f(z)=(z-$ $\left.z_{0}\right)^{n} g(z)=\left(z-z_{0}\right)^{m} h(z)$, where $h\left(z_{0}\right) \neq 0$. If $m>n$, then we may divide by $\left(z-z_{0}\right)^{n}$ to see that $g(z)=\left(z-z_{0}\right)^{m-n} h(z)$ and letting $z \rightarrow z_{0}$ yields $g\left(z_{0}\right)=0$, a contradiction. If $m<n$ a similar argument gives $h\left(z_{0}\right)=0$, which is also a contradiction. We conclude that $m=n$, thus $h=g$, and the theorem is proved.

Theorem 7. (The Argument Principle) Suppose $f(z)$ is meromorphic in an open set containing a circle $C$ and its interior. If $f$ has no poles and never 
vanishes on $C$, then $\frac{1}{2 \pi i} \int_{C} \frac{f^{\prime}(z)}{f(z)} d z=$ (number of zeros of $f(z)$ inside $C$ ) minus (number of poles of $f(z)$ inside $C$ ), where the zeros and poles are counted with their multiplicities.

(no proof given here, see, [2], p.90)

\subsection{The Riemann Zeta Functional Equations}

We know the gamma function $\Gamma(s)$ be defined for $s \in C$ with $\operatorname{Re}(s)>0$ by the integral

$$
\Gamma(s)=\int_{0}^{\infty} e^{-t} t^{s} \frac{d t}{t} .
$$

It satisfies a functional equation valid for all $s$ :

$$
\Gamma(s) \Gamma(1-s)=\frac{\pi}{\sin (\pi s)} \neq 0,
$$

and $\Gamma(s+1)=s \Gamma(s)$, which enables one to continue $\Gamma(s)$ analytically onto all of the complex $s$-plane, except that it has simple poles $s=0,-1,-2,-3, \ldots$ Using the two functional equations we easily see the reciprocal of $\Gamma(s)$ is an entire function of $s$. The identity reveals the symmetry of $\Gamma$ about the line $\operatorname{Re}(s)=\frac{1}{2}$, and the functional equation for $\zeta(s)$ it has a certain symmetry about the critical line $\operatorname{Re}(s)=\frac{1}{2}$, which is the relation

$$
\zeta(s)=2^{s} \pi^{s-1} \sin \left(\frac{1}{2} \pi s\right) \Gamma(1-s) \zeta(1-s)
$$

We know the function equation (1) shows that all the nontrivial zeros must lie in the strip $0<\operatorname{Re}(s)<1$, the so-called "critical strip". Note that $\zeta(s)$ has a certain symmetry about the real axis, namely

$$
\zeta(\bar{s})=\overline{\zeta(s)}
$$

They are easy to show that the nontrivial zeros are symmetrically located about the two lines. It follows that if $s_{0}$ is a complex number where $\zeta(s)$ has a zero of order $m$, then the complex conjugate $\overline{s_{0}}$ is a complex number where $\zeta(s)$ has a zero of the same order $m$, then $1-s_{0}$ is also a complex number where $\zeta(s)$ has a zero of the same order $m$, so is the complex conjugate $\overline{1-s_{0}}$, and therefore these orders $m$ are the same (which may be a pole, in which case $m$ is negative, but $\zeta(s)$ has no a pole in the region $0<\operatorname{Re}(s)<1)$. Indeed, by Theorem 6 and the analyticity of $\zeta$, when $\zeta$ has a zero of order $m$ at $s_{0}$, we can write

$$
\zeta(s)=\left(s-s_{0}\right)^{m} \varphi(s)
$$


in which case $m$ is a unique positive integer, where $\varphi(s)$ is analytic and nowhere vanishing in a neighborhood $U_{\rho_{0}}\left(s_{0}\right)$ of $s_{0}: U_{\rho_{0}}=\left\{s:\left|s-s_{0}\right|<\rho_{0}\right\}$ is contained in $0<\operatorname{Re}(s)<1$, and therefore

$$
\frac{\zeta^{\prime}(s)}{\zeta(s)}=\frac{m}{s-s_{0}}+G(s)
$$

where

$$
G(s)=\frac{\varphi^{\prime}(s)}{\varphi(s)},
$$

and the following $m$ is also the same positive integer. The conclusion is that if $\zeta$ has a zero of order $m$ at $s_{0}$, then $\zeta^{\prime} / \zeta$ has a simple pole with residue $m$ at $s_{0}$.

By the functional equations (1) and (2), we know the relations

$$
\zeta(s)=2^{s} \pi^{s-1} \sin \left(\frac{1}{2} \pi s\right) \Gamma(1-s) \zeta(1-s)=H(s) \zeta(1-s),
$$

and $\zeta(\bar{s})=\overline{\zeta(s)} \Leftrightarrow \zeta(s)=\overline{\zeta(\bar{s})}$ for $0<\operatorname{Re}(s)<1$, where $H(s)$ abbreviates the expression $2^{s} \pi^{s-1} \sin \left(\frac{1}{2} \pi s\right) \Gamma(1-s)$, and we can easily conclude that $H(s)$ is analytic and nowhere vanishing for $0<\operatorname{Re}(s)<1$. From (3) and the above relations we get

$$
\zeta(s)=H(s) \zeta(1-s)=\left(s-s_{0}\right)^{m} \varphi(s)=\overline{\zeta(\bar{s})},
$$

with $H(s) \neq 0$ and $\varphi(s) \neq 0$.

We proceed in two steps to give the further proof details as follows.

Proof. First,we obviously observe that $\zeta(1-s)=\left(s-s_{0}\right)^{m} \cdot \frac{\varphi(s)}{H(s)}$. Substituting $s=1-z$, we get the Eq.(7):

$$
\begin{gathered}
\zeta(z)=\left(1-z-s_{0}\right)^{m} \cdot \varphi(1-z) / H(1-z), \\
\zeta(z)=\left[-\left(z-\left(1-s_{0}\right)\right)\right]^{m} \cdot \varphi(1-z) / H(1-z), \\
\zeta(z)=\left(z-\left(1-s_{0}\right)\right)^{m} \cdot(-1)^{m} \varphi(1-z) / H(1-z), \\
\zeta(z)=\left(z-\left(1-s_{0}\right)\right)^{m} g(z),
\end{gathered}
$$

where $g(z)$ abbreviates the expression $(-1)^{m} \varphi(1-z) / H(1-z)$, and $g(z)$ is also analytic and nowhere vanishing for $0<\operatorname{Re}(z)<1$, and because $z$ is any complex variable in the region $0<\operatorname{Re}(z)<1$, we can make the substitution $z=s$ in the desired Eq.(7), we have

$$
\zeta(s)=\left(s-\left(1-s_{0}\right)\right)^{m} g(s)
$$


where $g(s)$ is analytic and nowhere vanishing in a neighborhood $U_{\rho_{1}}\left(1-s_{0}\right)$ of $\left(1-s_{0}\right): U_{\rho_{1}}\left(1-s_{0}\right)=\left\{s:\left|s-\left(1-s_{0}\right)\right|<\rho_{1}\right\}$ is contained in $0<\operatorname{Re}(s)<1$, and therefore

$$
\frac{\zeta^{\prime}(s)}{\zeta(s)}=\frac{m}{s-\left(1-s_{0}\right)}+h(s)
$$

where $h(s)=g^{\prime}(s) / g(s)$. The conclusion is that if $\zeta$ has a zero of order $m$ at $s_{0}$, then $\zeta$ also has a zero of the same order $m$ at $1-s_{0}$. By Theorem 6 and the argument principle we have

$$
\frac{1}{2 \pi i} \int_{C_{1}} \frac{\zeta^{\prime}(s)}{\zeta(s)} d s=\frac{1}{2 \pi i} \int_{C_{2}} \frac{\zeta^{\prime}(s)}{\zeta(s)} d s=m,
$$

in which case $m$ is a positive integer, being $\zeta(s)$ has no a pole in the region $0<$ $\operatorname{Re}(s)<1$, and where the $C_{1}$ is any closed contour that it can be continuously deformed into any circle in the domain which is in a neighborhood $U_{\rho_{0}}\left(s_{0}\right)$ of $s_{0}: U_{\rho_{0}}\left(s_{0}\right)=\left\{s:\left|s-s_{0}\right|<\rho_{0}\right\}$ contained in $0<\operatorname{Re}(s)<1$, where the $C_{2}$ is any closed contour that it can be continuously deformed into any circle in the domain, which is in a neighborhood $U_{\rho_{1}}\left(1-s_{0}\right)$ of $1-s_{0}: U_{\rho_{1}}\left(1-s_{0}\right)=$ $\left\{s:\left|s-\left(1-s_{0}\right)\right|<\rho_{1}\right\}$ contained in $0<\operatorname{Re}(s)<1$.

We usually write $s_{0}=\sigma_{0}+i t_{0}, \phi(s)=-\zeta^{\prime} / \zeta(s)$, where $\sigma_{0}$ and $t_{0}$ are the real numbers. The following two limits follow immediately from the above results in this first step:

$$
\begin{aligned}
& \lim _{s \rightarrow s_{0}}\left(s-s_{0}\right) \phi(s)=-m, \\
& \lim _{s \rightarrow s_{1}}\left(s-s_{1}\right) \phi(s)=-m,
\end{aligned}
$$

where $s_{1}=1-s_{0}$, in which case $m$ is a positive integer. By the formula $\zeta(\bar{s})=\overline{\zeta(s)}$, we can observe and conclude that a similar fact also holds if $\zeta$ has a zero of order $m$ at $1-s_{0}$, then $\zeta$ also has a zero of the same order $m$ at the complex conjugate $\overline{1-s_{0}}$.

Second, by Theorem 6 and the argument principle, being $\zeta$ has a zero of order $m$ at $s_{0}$, we can write

$$
\overline{\zeta(s)}=\overline{\left(s-s_{0}\right)^{m} \cdot \varphi(s)}=\left(\overline{s-s_{0}}\right)^{m} \cdot \overline{\varphi(s)}=\left(\bar{s}-\overline{s_{0}}\right)^{m} \cdot \overline{\varphi(s)} .
$$

Substituting $\bar{s}=z$, and so $s=\bar{z}$, also $\zeta(\bar{s})=\overline{\zeta(s)}$, we get

$$
\zeta(\bar{s})=\overline{\zeta(s)}=\left(z-\overline{s_{0}}\right)^{m} \overline{\varphi(\bar{z})}=\left(z-\overline{s_{0}}\right)^{m} f(z),
$$

where $f(z)$ abbreviates the expression $\overline{\varphi(\bar{z})}$, and $f(z)$ is analytic and nowhere vanishing for $0<\operatorname{Re}(z)<1, \varphi(s)$ is analytic and so is $\overline{\varphi(s)}$ by the complex differentiability. Therefore

$$
\zeta(z)=\left(z-\overline{s_{0}}\right)^{m} f(z)
$$


Making the substitution $z=s$ in the desired Eq.(11), we have

$$
\zeta(s)=\left(s-\overline{s_{0}}\right)^{m} f(s)
$$

where $f(s)$ is analytic and nowhere vanishing in a neighborhood $U_{\rho_{2}}\left(\overline{s_{0}}\right)$ of $\overline{s_{0}}$ : $U_{\rho_{2}}\left(\overline{s_{0}}\right)=\left\{s:\left|s-\overline{s_{0}}\right|<\rho_{2}\right\}$ is contained in $0<\operatorname{Re}(s)<1$, and therefore

$$
\frac{\zeta^{\prime}(s)}{\zeta(s)}=\frac{m}{s-\overline{s_{0}}}+R(s)
$$

where $R(s)=f^{\prime}(s) / f(s)$. The case can be carried out similarly. By Theorem 6 and the argument principle, we have

$$
\frac{1}{2 \pi i} \int_{C_{2}} \frac{\zeta^{\prime}(s)}{\zeta(s)} d s=\frac{1}{2 \pi i} \int_{C_{3}} \frac{\zeta^{\prime}(s)}{\zeta(s)} d s=m,
$$

in which case $m$ is a positive integer, being $\zeta(s)$ has no a pole in the region $0<\operatorname{Re}(s)<1$, and where the $C_{2}$ is the same one as previously, and the $C_{3}$ is any closed contour that it can be continuously deformed into any circle in the domain which is in a neighborhood $U_{\rho_{3}}\left(\overline{1-s_{0}}\right)$ of $\overline{1-s_{0}}: U_{\rho_{3}}\left(\overline{1-s_{0}}\right)=$ $\left\{s:\left|s-\left(\overline{1-s_{0}}\right)\right|<\rho_{3}\right\}$ is also contained in $0<R e(s)<1$.

The following two limits follow immediately from the above results in this second step:

$$
\begin{aligned}
& \lim _{s \rightarrow s_{1}}\left(s-s_{1}\right) \phi(s)=-m, \\
& \lim _{s \rightarrow s_{2}}\left(s-s_{2}\right) \phi(s)=-m,
\end{aligned}
$$

where $s_{1}=1-s_{0}, s_{2}=\overline{1-s_{0}}$, whence our three limits (9),(13), and (14) are just equal to the same $-m$ from the above justification, which are just the same as the residue $-m$ of $-\zeta^{\prime} / \zeta$ defined by $\phi$ in the same domain regarding a neighborhood of the zero of $\zeta$, in which case $s_{0}$ is equal to a parameter $\sigma_{0}+i t_{0}$, and in which case $m$ is a positive integer as above.

Now the following formulas (15) and (16) are trivially verified by the formulas (9) and (14):

$$
\begin{gathered}
\lim _{\sigma \rightarrow \sigma_{0}}\left(\sigma-\sigma_{0}\right) \phi\left(\sigma+i t_{0}\right)=-m, \\
\lim _{\sigma \rightarrow \sigma_{0}}\left(\sigma-1+\sigma_{0}\right) \phi\left(\sigma+i t_{0}\right)=-m .
\end{gathered}
$$

We usually write $s_{0}=\sigma_{0}+i t_{0}, \phi(s)=-\zeta^{\prime} / \zeta(s)$, where $\sigma_{0}$ and $t_{0}$ are the real numbers. Obviously, (15) and (16) have the same variable $\sigma$ in the same domain 
of the definition of $\phi$.

Therefore

$$
\lim _{\sigma \rightarrow \sigma_{0}}\left(\sigma-\sigma_{0}\right) \phi\left(\sigma+i t_{0}\right)=-m
$$

which is the same as

$$
\lim _{\sigma \rightarrow \sigma_{0}}\left(\sigma-1+\sigma_{0}\right) \phi\left(\sigma+i t_{0}\right)=-m
$$

\subsection{Conclusions by Some Limits}

Then by (15) and (16) we get

$$
\lim _{\sigma \rightarrow \sigma_{0}}\left(\sigma_{0}-\frac{1}{2}\right) \phi\left(\sigma+i t_{0}\right)=0
$$

namely

$$
\left(\sigma_{0}-\frac{1}{2}\right) \lim _{\sigma \rightarrow \sigma_{0}} \phi\left(\sigma+i t_{0}\right)=0,
$$

then $\sigma_{0}=\frac{1}{2}$ or $\lim _{\sigma \rightarrow \sigma_{0}} \phi\left(\sigma+i t_{0}\right)=0$, also $\lim _{\sigma \rightarrow \sigma_{0}}\left(\sigma-\sigma_{0}\right)=0$, and therefore, if

$$
\lim _{\sigma \rightarrow \sigma_{0}} \phi\left(\sigma+i t_{0}\right)=0
$$

then

$$
\lim _{\sigma \rightarrow \sigma_{0}}\left(\sigma-\sigma_{0}\right) \phi\left(\sigma+i t_{0}\right)=-m=0 .
$$

So, either $\sigma_{0}=\frac{1}{2}$ or $m=0$ what we want to get. But using Theorem 6 and the argument principle, with being $\zeta(s)$ has no a pole in the region $0<\operatorname{Re}(s)<1$, then we know that the above $m$ is a unique positive integer, so we have $m \neq 0$. Therefore, we only get the one $\sigma_{0}=\frac{1}{2}$. Hence it can be seen that all the nontrivial zeros of $\zeta(s)$ in the critical strip lie on the line $\operatorname{Re}(s)=\frac{1}{2}$. This gives us a proof of the Riemann Hypothesis.

\section{The Third Proof from Topological Perspective}

\subsection{The Notion for Possibly Using}

Definition 8. (Homeomorphism) A bijective map $f: X \rightarrow Y$ is called a homeomorphism when $f$ and $f^{-1}$ are continuous, that is when $U \subset X$ is open if and only if $f(U) \subset Y$ is. 
Definition 9. (Hausdorff Separation Axiom) A topological space is called Hausdorff if for any two different points there exist disjoint neighborhoods.

Theorem 10. A continuous bijection $f: X \rightarrow Y$ from a compact space $X$ into a Hausdorff space $Y$ is always a homeomorphism. (see,[4], p.23)

Theorem 11. The interior and boundary of surface are disjoint. (see [3], p.116)

Theorem 12. Homeomorphic surfaces have homeomorphic boundaries. (see [3], p.117)

Definition 13. (Contractible Space) A topological space is called contractible if it is equivalent to a space with one point.It is a simple but important special case for our applications.

\subsection{Recalling the Riemann Zeta Functional Equations}

The functional equation for $\zeta(s)$ is the relation it has a certain symmetry about the critical line $\operatorname{Re}(s)=\frac{1}{2}$, namely

$$
\zeta(s)=2^{s} \pi^{s-1} \sin \left(\frac{1}{2} \pi s\right) \Gamma(1-s) \zeta(1-s)
$$

Note that $\zeta(s)$ has a certain symmetry about the real axis, namely

$$
\zeta(\bar{s})=\overline{\zeta(s)}
$$

All the nontrivial zeros of $\zeta(s)$ in the critical strip lie on the line $R e(s)=\frac{1}{2}$, which is called the Riemann Hypothesis.

The two function equations shows that all the nontrivial zeros must lie in the strip $0<\operatorname{Re}(s)<1$, the so-called "critical strip". It is easy to show that the zeros are symmetrically located about the real axis and about the "critical line" $R e(s)=\frac{1}{2}$. It follows that if $s_{0}$ is a complex number where $\zeta(s)$ has a zero of order $m$, then the complex conjugate $\overline{s_{0}}$ is a complex number where $\zeta(s)$ has a zero of the same order $m$, then $1-s_{0}$ is also a complex number where $\zeta(s)$ has a zero of the same order $m$, so is the complex conjugate $\overline{1-s_{0}}$ and therefore these orders $m$ are the same (which may be a pole, in which case $m$ is negative, but $\zeta(s)$ has no a pole in the region $0<\operatorname{Re}(s)<1)$.

\subsection{The Boundary of a Surface}

We have developed enough machinery to say exactly what we mean by a surface, and free ourselves from the straight jacket of having to work inside some 
Euclidean space.We see every metric space is Hausdorff, any Euclidean space with the usual distance between points is a metric space, e.g., an unit circle in the plane, an $\varepsilon$-ball, a disc $D^{2}$, a square, a rectangle, a sphere, a torus,etc. One sees that sets $\left\{x_{i}\right\}$ consisting of a single point $x_{i}$ is compact, which it is of course closed and bounded.

A surface (sometimes which may be without an edge or boundary) is a topological space in which each point has a neighborhood homeomorphic to the plane $E^{2}$, and for which any two distinct points possess disjoint neighborhood. We have given the simplest possible definitions. If we wish to allow a surface to have an edge or boundary (as in the case of the Möbius strip), then we cannot expect every point to have a neighborhood homeomorphic to the plane $E^{2}$. We must allow in addition points which have neighborhoods homeomorphic to the upper half-plane $E_{+}^{2}$ (consisting of those points of the plane whose $y$-coordinates are greater than or equal to zero). All of our examples of surfaces now fit in nice with this definition when they are given the subspace topology from Euclidean space.

A surface is a Hausdorff space $S$ in which each point has a neighborhood homeomorphic either to the plane $E^{2}$, or to the closed half space $E_{+}^{2}$. The interior of $S$ consists of the points of $S$ which have a neighborhood homeomorphic to $E^{2}$. Those points $z \in S$ for which there is an open neighborhood $U$, and a homeomorphism $f: U \rightarrow E_{+}^{2}$ such that $f(z)=0$, form the boundary of $S$, the points on the boundary of $z$ named boundary points.

We already know that the points on the surface, if it has no an open neighborhood homeomorphic to $E^{2}$. Of course, it has an open neighborhood homeomorphic to $E_{+}^{2}$, those points are called a boundary point. We also know $E_{+}^{2}$ is not homeomorphic to $E^{2}$. For disc $D^{2}$, the points on the bounding circle $S^{1}$ are a boundary point,those points have an open neighborhood homeomorphic to $E_{+}^{2}$, will there still be an open neighborhood homeomorphic to $E^{2}$ ? These definitions satisfy our intuition as to what "interior" and "boundary" should mean for a surface. We must check that a point cannot lie both in the interior and on the boundary.

Theorem 14. Let $z$ be a point on the surface $S$, and $V \subset S$ is an open neighborhood of $z$ and a homeomorphic map $f: V \rightarrow E_{+}^{2}$ such that $f(z)=0$, then the point $z$ has no an open neighborhood homeomorphic to $E^{2}$.

Proof. We shall assume the result false and obtain a contradiction. Suppose $z$ has a neighborhood $U$ homeomorphic to $E^{2}$, and $g: U \rightarrow E^{2}$ is a homeomorphic map, this means we can find an open neighborhood $f(U \cap V)$ of the origin $O=f(z)$ in $E_{+}^{2}$ is homeomorphic to an open neighborhood $g(U \cap V)$ 
of the point in $E^{2}$. Choose an $\varepsilon$-open ball $B(O, \varepsilon)$ in $E_{+}^{2}$ with $\varepsilon>0$, center the origin and small enough so that $B(O, \varepsilon) \subset f(U \cap V) \subseteq E_{+}^{2}$. Set a point $x \in W \subseteq g(U \cap V)$. By our assumption, because $f$ and $g$ are homeomorphisms, we know that $B(O, \varepsilon)$ is homeomorphic to an open neighborhood $W$ of $x$ in $E^{2}$. Then $B(O, \varepsilon)-\{O\}$ is homeomorphic to $W-\{O\}$, but the former $B(O, \varepsilon)-\{O\}$ is easily seen to be contractible, and the latter $W-\{O\}$ is not contractible. This gives us our contradiction. Thus proving this theorem.

\subsection{Some Basic Properties of A Rectangle}

A rectangle of course is a surface, a rectangle has a boundary, a rectangle is homeomorphic to a disc $D^{2}$, a rectangle is a compact space and a Hausdorff Space. A rectangle is always contained in a plane. A rectangle is closed in the plane. The boundary points of a rectangle have an open neighborhood homeomorphic to $E_{+}^{2}$, so have its four vertexes being also a boundary point.

\subsection{Using Topological Viewpoint Justifies the Riemann Hypothesis}

Proof. From now on, we can then apply Theorem 14, whose proof applies to the zeta function $\zeta(s)$ satisfying our conditions: a point $s=z$ on the surface $S$ which it can be a subset of the plane, $V \subset S$ is an open neighborhood of $z$ and a homeomorphic map $f: V \rightarrow E_{+}^{2}$ such that $f(z)=0$. By Theorem 14, we can conclude that all nontrivial zeros of the zeta function $\zeta(s)$ have no an open neighborhood homeomorphic to $E^{2}$, whereas all nontrivial zeros of the zeta function $\zeta(s)$ have a certain symmetry about the real axis and the line $R e(s)=\frac{1}{2}$, namely $\zeta(\bar{s})=\overline{\zeta(s)}$ and $\zeta(s)=2^{s} \pi^{s-1} \sin \left(\frac{1}{2} \pi s\right) \Gamma(1-s) \zeta(1-s)$. The conclusion follows that $\zeta$ has a zero of order $m$ at $s_{0}$, then $\zeta$ also has a zero of the same order $m$ at the complex conjugate $\overline{s_{0}}$. It also follows that $\zeta$ has a zero of the same order $m$ at $1-s_{0}$, then $\zeta$ also has a zero of the same order $m$ at the complex conjugate $\overline{1-s_{0}}$. It is easily seen to know that if we assume $s_{0} \neq \overline{1-s_{0}}$, then there is a rectangle with four vertexes consisting of the four different points $x_{1}=s_{0}, x_{2}=\overline{s_{0}}, x_{3}=1-s_{0}$, and $x_{4}=\overline{1-s_{0}}$. Let $x_{i}$ are the four vertexes of the rectangle, choose four open discs (i.e., an $\varepsilon$-open ball in $\left.E^{2}\right): B\left(x_{i}, \varepsilon\right), i=1,2,3,4$, centered at the point $x_{i}$ and $\varepsilon$ is small enough so that $U=\bigcup_{i=1}^{4} B\left(x_{i}, \varepsilon\right)$, then it is an open neighborhood of $x_{i}$ contained in the rectangle. Obviously, $U$ is homeomorphic to $E^{2}$. One sees immediately that $U=\bigcup_{i=1}^{4} B\left(x_{i}, \varepsilon\right)$ which is an open neighborhood of $x_{i}$. But using Theorem 14, we know that if $\zeta\left(x_{i}\right)=0$, then $x_{i}$ is a boundary point in the rectangle, which has no an open neighborhood of $x_{i}$ homeomorphic 
to $E^{2}$, but $U$ is homeomorphic to $E^{2}$, which is a contradiction. Then there isn't any rectangle with the four vertexes contained in the plane, and then our assumption $s_{0} \neq \overline{1-s_{0}}$ is false and obtain a contradiction. Thus we have $s_{0}=\overline{1-s_{0}}$. We usually write $s_{0}=\sigma_{0}+i t_{0}$, where $\sigma_{0}$ and $t_{0}$ are the real numbers. Furthermore,we get $s_{0}=\sigma_{0}+i t_{0}=\overline{1-s_{0}}=\overline{1-\left(\sigma_{0}+i t_{0}\right)}=1-\sigma_{0}+i t_{0}$, and therefore we have $\sigma_{0}=\frac{1}{2}$. This also proves the Riemann Hypothesis.

\section{Acknowledgments}

The author would like to thank the experts for their invaluable comments and encouragements regarding this article, and especially wants to thank the readers of International Journal of Pure and Applied Mathematics for making the journal successful. Finally, the author thanks the editorial team of IJPAM.

\section{References}

[1] Serge Lang, Complex Analysis, 4-th Edition, Springer-Verlag and Beijing World Publishing Corporation, Beijing (2003).

[2] Elias M. Stein, Rami Shakarchi, Complex Analysis, Princeton University Press and Beijing World Publishing Corporation, Beijing (2007).

[3] M.A. Armstrong, Basic Topology, Springer-Verlag and Beijing World Publishing Corporation, Beijing (2008).

[4] Klaus Jänich, Topology, Springer-Verlag and Beijing World Publishing Corporation, Beijing (2012).

[5] Hao-Cong Wu, Showing how to imply proving the Riemann hypothesis, European J. Math. Sci., 2, No. 2 (2013), 126-139. 
